\title{
The spectrum of the roAp star HD 101065 (Przybylski's star) in the Li I $6708 \AA$ spectral region ${ }^{\star}$
}

\author{
A. V. Shavrina ${ }^{1}$, N. S. Polosukhina ${ }^{2,10}$, Ya. V. Pavlenko ${ }^{1}$, A. V. Yushchenko ${ }^{3,9}$, P. Quinet ${ }^{4,5}$, M. Hack $^{6}$, P. North $^{7}$, \\ V. F. Gopka ${ }^{3}$, J. Zverko ${ }^{8}$, J. Žhižnovský ${ }^{8}$, and A. Veles ${ }^{1}$
}

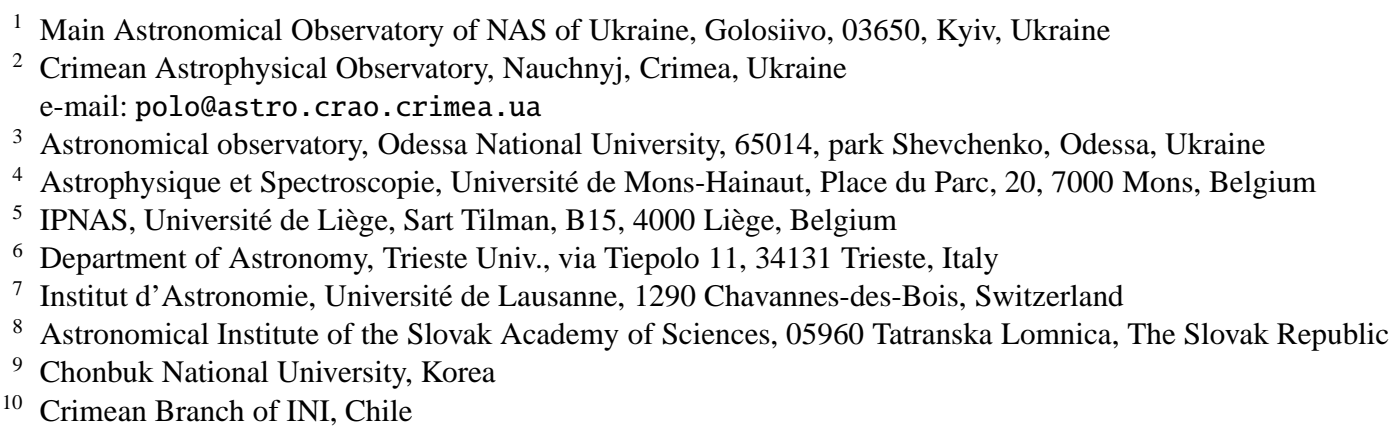

Received 18 November 2002 / Accepted 1 July 2003

\begin{abstract}
We carried out a detailed analysis of spectra of the unique roAp star HD 101065 (Przybylski's star) near the resonance doublet Li I $6708 \AA$ A, using a most complete line list including all possible transitions between REE levels of the NIST database. Our model calculations were performed under two assumptions: a blend of Li and REE lines, and a blend of REE lines only. They prove that Li lines are present in the range 6707.72-6708.02 $\AA$ and that the resulting Li abundance is 3.1 dex (in the scale $\log N(\mathrm{H})=12.0$ ), while the isotopic ratio ${ }^{6} \mathrm{Li} /{ }^{7} \mathrm{Li}$ is near to 0.3 .
\end{abstract}

Key words. stars: chemically peculiar - stars: individual: HD 101065

\section{Introduction}

Among the rapidly oscillating Ap (roAp hereafter) stars, HD 101065 is the most unusual (Przybylski 1961, 1966). The report of Przybylski in 1976 at the IAU Symp. 32, showing the very specific properties of the spectrum of HD 101065, made a very strong impression on specialists of Ap stars and at once attracted much attention to this star. Significant differences between the spectrum of this star and the spectra of other Ap stars were pointed out by Przybylski: first, the absence or extreme weakness of lines of elements that appear generally normal or close to normal in other Ap stars, like iron and some other iron group elements, then the abnormal strengthening of Rare Earth Elements (REE hereafter) lines. Przybylski predicted the presence of a magnetic field for this star. He proposed to explain the observed anomalies of elemental abundances by the stability of the upper atmosphere due to a strong magnetic field, which would favour radiative diffusion. Wolff \& Hagen (1976) did find a magnetic field of about $2.5 \mathrm{KG}$ in this mysterious

Send offprint requests to: A. V. Shavrina,

e-mail: shavrina@mao.kiev.ua

* Based on observations collected at the European Southern Observatory, La Silla, Chile (programme 56.E-0640). star. Kurtz \& Wegner (1979) began photometric observations of HD 101065 in the seventies and detected well-defined pulsations with a peak-to-peak amplitude of about $0.012 \mathrm{mag}$ and a period of $12.14 \mathrm{~min}$.

It was the first Ap star in which pulsations were found. Now this phenomenon is known to be typical of other Ap stars, which form the group of roAp stars. The first attempts to identify the lines in the spectrum of HD 101065 were made by Przybylski (1961, 1966), and Warner (1966). These papers mention for the first time the possible presence of the resonance lithium doublet. In the subsequent study of this star, Wegner \& Petford (1974) have proposed that it is the coolest of Ap stars. They considered the very strange spectrum to be the combined result of low temperature and high surface REE abundances (the abundance excess is about 4 dex). Beginning with Przybylski, investigators suggested that heavy line blanketing changes the atmosphere structure very strongly, and that the computation of a realistic model atmosphere for this star is complicated by the need to take REE line blanketing into account. Cowley et al. $(1998,2000)$ examined in detail the problem of effective temperature to define the model atmosphere of HD 101065, and have carried out a thorough quantitative analysis (abundances of 54 elements) of the spectrum in the range 
3959-6652 ̊. Cowley \& Mathys (1998) have noted in their conclusion that the presence of the third spectrum REE (Pr III, $\mathrm{Nd}$ III, Ce III...) is evidence for the unusual structure of the atmosphere of this star, which includes a very thin surface convective zone typical of stars of spectral type F2. One possible explanation of these extreme abundance anomalies is radiative diffusion, allowed by the stabilization of the atmosphere by a strong magnetic field. Cowley et al. (1998, 2000) did not address the question of the lithium abundance in the atmosphere of this star. The present work is a further investigation of the spectrum of HD 101065 in the region $6675-6735 \AA$; the main goal is the analysis of the lithium blend $6708 \AA$ and estimation of the atmospheric lithium abundance.

The very complicated spectral feature at $\lambda 6708 \AA$ in the observed spectra raises the problem of correct line identification, especially REE lines, in this region. A comprehensive analysis of REE lines with the latest atomic data was performed, and we show that the resonance doublet of lithium is the main contributor to the $6708 \AA$ feature.

\section{Lithium in the spectrum HD 101065}

The presence of lithium in the spectrum of this unique star was first mentioned by Przybylski (1961), and this remark was noticed by Warner (1966), who made additional observations of this star at the Radcliffe Observatory, using the $74^{\prime \prime}$ reflector, in the spectral range 3770-6880 $\AA$ (dispersion $6 \AA / \mathrm{mm}$ ). He found very strong lines of singly ionized REE in the region 5500-6880 $\AA$, the relative intensities of which are similar to laboratory intensities from the tables of Meggers et al. (1961). However, as mentioned by Przybylski (1966), the line Sm II $6707.45 \AA$ can be considered only as part of the lithium blend, and the main contribution to this blend remains the resonant lithium doublet Li I $6708 \AA$. The first estimation of the lithium abundance relative to the solar value was made by Warner (1966): [Li] = 2.4 dex. This author also mentioned the probable presence of ${ }^{6} \mathrm{Li}$.

\section{Observations and reduction}

The observations were made by one of us (PN) with the $1.4 \mathrm{~m} \mathrm{CAT}$ at ESO La Silla in March 1996, in the framework of the International Project "Lithium in magnetic CP stars". The Coudé Échelle Spectrograph was used with resolving power $R=100000$ and $S / N \geq 100$. The usual data reduction (bias and dark subtraction, division by flat-field, extraction and wavelength calibration) was done by PN during the observing run, using the old IHAP system of ESO (see North et al. 1998 for more details). In Table 1 we give the epoch, exposure time and wavelength coverage of our observations.

\section{Model atmosphere}

We have computed a model atmosphere with $T_{\text {eff }}=6600 \mathrm{~K}$, $\log g=4.2$ and abundances of most (54) elements from Cowley et al. (2000). The other element abundances were adopted from Kurucz (1999). A detailed discussion of model atmosphere
Table 1. List of observations for HD 101065.

\begin{tabular}{rrrccc}
\hline \hline & $\begin{array}{r}\text { Date } \\
\text { d.m.y }\end{array}$ & $\begin{array}{r}\text { UT } \\
\text { h } \mathrm{m}\end{array}$ & $\begin{array}{r}\text { Exp. } \\
{[\mathrm{m}]}\end{array}$ & $\begin{array}{c}\text { HJD } \\
2450000+\end{array}$ & Range \\
\hline 04 & 11.3 .96 & 511 & 20 & 153.726 & $6675-6735 \AA$ \\
26 & 10.3 .96 & 441 & 20 & 152.705 & $6120-6180 \AA$ \\
\hline
\end{tabular}

parameters for HD 101065 can be found in the abovementioned paper, as well as in the paper by Piskunov \& Kupka (2001).

The most recent method for determining fundamental stellar parameters through pulsation analysis was applied by Matthews et al. (1999) to some roAp stars, including HD 101065. But it needs a photometric calibration. $\mathrm{H}_{\beta}$ calibration results in $T_{\text {eff }}=7400 \mathrm{~K}$, which is too high, as confirmed by detailed spectral analysis (see Cowley et al. 2000). We used the SAM12 program (see Pavlenko 2003a, 2003b), a modified version of Kurucz's code ATLAS12 (Kurucz 1999). The essential changes were made in the subroutines accounting for opacities. Namely, the opacity caused by absorption of atomic lines was taken into account with the Opacity Sampling (OS) technique (Sneden et al. 1976). Then, bound-free absorption crosssection of C I, N I and O I atoms in the wide spectral region 30-8880 nm were calculated by us, Pavlenko \& Zhukovskaya (2003), using the data of the TOPBASE project (Seaton 1992). New opacity tables of the bound-free absorption of C I, N I, O I atoms, reduced to the format of Hofsaess (1979) opacity tables, are available on the Pavlenko's web site (Pavlenko 2003c). The importance of $b f$ absorption for formation of spectra of different stars is discussed elsewhere (Pavlenko 1999, 2003a; Asplund et al. 2000).

The basic advantage of the SAM12 code is the possibility to calculate model atmospheres for stars with chemical composition different from the solar one, as well as in ATLAS12. Abundances of elements were set as input data which do not change with depth. The microturbulence velocity was set constant with depth.

The profile of the line absorption coefficient was defined by a Voigt function $H(a, v)$; the damping constants were taken from line databases or calculated within the frame of the Unsöld approximation (Unsöld 1955). The list of absorption lines of atoms and ions for the range $\lambda \lambda 40-60000 \mathrm{~nm}$ was compiled using the VALD database (Kupka et al. 1999); the REE lines data, calculated with Cowan's code (Quinet et al. 1999), are taken from the DREAM database (http: //www . umh . ac . be/ astro/dream . shtml). Unfortunately, this database contains lines of only about one quarter of all rare earth ions. Therefore, in the opacity sampling calculation we consider two samples of REE lines. In the first we calculated the absorption provided by the VALD and DREAM lines. Then, we amplified the absorption of DREAM lines by a factor of 10 (in addition to the VALD lines) to compensate for the incompleteness of the REE line list. The comparison of these two cases shows that REE line opacity does not affect significantly the temperature distribution in our model atmospheres. 


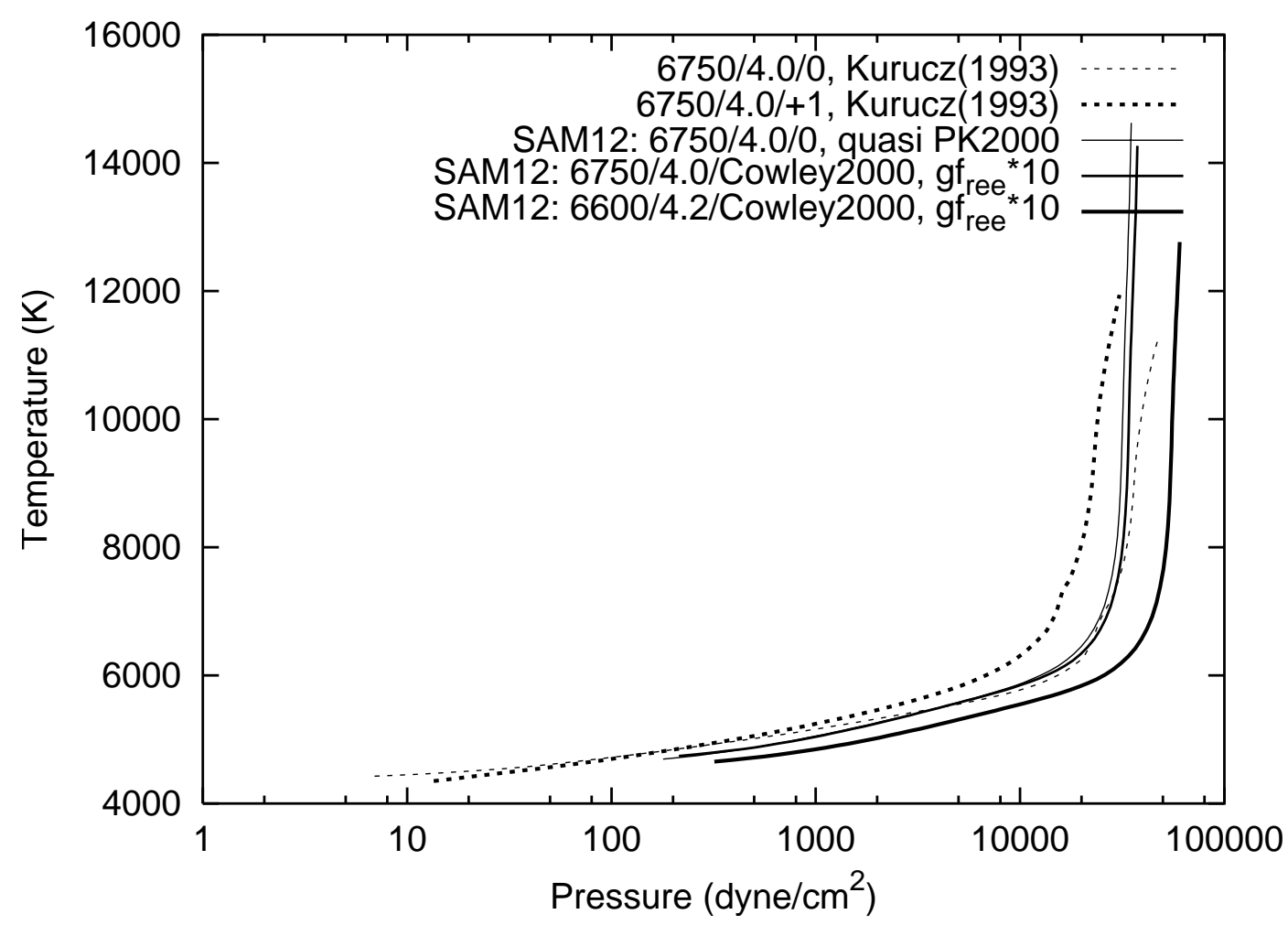

Fig. 1. $T-P$ relation for model atmospheres considered in this work. Two models from Kurucz's (1993) grid are show, as well as SAM12 simulation of model atmosphere using the Piskunov \& Kupka (2000) approach; SAM12 model atmosphere computed with Cowley (2000) abundances and REE $b f$ opacities enhanced by factor 10 of the same $T_{\text {eff }}$ and $\log g$; structure of model atmosphere $T_{\text {eff }}=6600 / 4.2$ with Cowley abundances and enhanced REE opacities is shown also. See text for more details.

Kurucz's models with $T_{\text {eff }}=6750 \mathrm{~K}, \log g=4.0$ (Kurucz 1999) and abundances of Cowley et al. (2000) were also used in calculations of synthetic spectra. We calculated $T_{\text {eff }} / \log g /[M]=6750 / 4.0 / 0$ model with solar abundances, as described previously, and compared them with Kurucz models (Kurucz 1993). Comparison of temperature structures and corresponding synthetic spectra shows their similarity. As our model atmosphere is rather iron deficient, the increase of opacity due to the REE elements may be compensated by the weakness of iron lines.

Cowley et al. analysed the spectrum of HD 101065 with the ATLAS-9 model with Opacity Distribution Function (ODF) tables, calculated by the authors on the basis of scaled solar abundances to fit better the observed color indices, in particular the unusually low $c_{1}$ index. The model atmosphere for this star was also computed by Piskunov \& Kupka (2001), also through the ODF method, with a 2.25 dex Fe overabundance and a 2.49 dex $\mathrm{Ca}$ excess to simulate unknown opacities in the ODF tables. These authors used the abundances of Cowley et al. (2000) for model computation with these ODF. We have followed this approach for the OS method, by computing line opacity due to the $\mathrm{Fe} \mathrm{I}$ and $\mathrm{Ca} \mathrm{I}$ lines with factors for gf- values of 177.8 (2.25 dex) and 308 (2.49 dex) respectively. In Fig. 1 we compare the $T-P$ relation for model atmospheres with $T_{\text {eff }}=6750 \mathrm{~K}$ and $\log g=4.0$ of Kurucz (solar abundance and $[M]=+1$ ) and ours with Cowley's abundances, calculated in two ways - the OS model, similar to the ODF model of Piskunov \& Kupka (SAM12:6750/4.0/0, analogue PK2000), and our two OS model atmospheres for $T_{\text {eff }}=6750$ and $6600 \mathrm{~K}$ and $\log g=4.0$, with $g f$ (REE) lines enhanced by a factor of 10 (SAM12:6750/4.0/0/Cowley 2000, $g f_{\text {ree }} * 10$ and SAM12:6600/4.2/0/Cowley 2000, $\left.g f_{\text {ree }} * 10\right)$. In general, the photospheres of models with higher opacities are shifted upwards, into the layers of lower pressure; however, the differences in the $T-P$ relation are rather small.

It is worth noting that our OS model atmospheres $6750 / 4.0 / 0$ and $6750 / 4.0 /+1$ agree with the corresponding Kurucz ODF models within $50 \mathrm{~K}$ in the line forming regions. Therefore, our OS model atmosphere with "metal rich" opacities should follow the Piskunov \& Kupka (2001) ODF model.

To analyze the Li blend, we used an atmosphere model with $T_{\text {eff }}=6600 \mathrm{~K}, \log g=4.2$. The resulting abundances were compared for this model and for the OS model, which is similar to that of Piskunov \& Kupka for the same parameters, both for Cowley's abundances, and for Kurucz's model for solar abundances. They are cited and discussed in the next section.

\section{Li blend analysis and REE line contribution to the 6705.75-6708.75 A spectral region}

We calculated the synthetic spectra in the range 6705.75-6708.75 $\AA$ with the help of the STARSP (Tsymbal 1996) and ROTATE codes (see Shavrina et al. 2000), both developed by V. Tsymbal, and of the URAN code (Yushchenko 1997). All cited codes are based on Kurucz' modules for synthetic spectra ("SYNTHE", 1993). The URAN program 
Table 2. The list of lines used for spectra calculations in the range 6705.75-6708.75 $\AA$.

\begin{tabular}{|c|c|c|c|c|c|c|c|c|c|}
\hline El. & $\lambda, \AA$ & $\overline{E^{\prime \prime}}$ & $\overline{\log g f}$ & Source & El. & $\lambda, \AA$ & 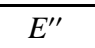 & $\overline{l \log g f}$ & Source \\
\hline *Dy II & 6705.727 & 2.078 & -2.68 & & ${ }^{*}$ Gd II & 6707.462 & 3.270 & -1.98 & \\
\hline$* \mathrm{Nd}$ II & 6705.891 & 3.269 & -2.70 & & Sm II & 6707.473 & 0.930 & -1.48 & VALD \\
\hline $\mathrm{Yb}$ II & 6705.965 & 5.856 & -3.04 & DREAM & $\mathrm{Yb}$ II & 6707.603 & 6.651 & -1.38 & DREAM \\
\hline Ce II & 6706.051 & 1.840 & -0.95 & DREAM & $*$ Sm II & 6707.648 & 1.746 & -1.27 & \\
\hline Tm II & 6706.150 & 5.322 & -1.07 & DREAM & Nd II & 6707.755 & 0.170 & -3.55 & DREAM[Quinet] \\
\hline Tm II & 6706.262 & 3.955 & -2.36 & DREAM & ${ }^{7} \mathrm{Li} \mathrm{I}$ & 6707.756 & 0.000 & -0.427 & [Smith et al.] \\
\hline Ce II & 6706.307 & 3.195 & -2.40 & DREAM & ${ }^{7} \mathrm{Li} \mathrm{I}$ & 6707.768 & 0.000 & -0.206 & -"- \\
\hline *Pr III & 6706.492 & 3.104 & -1.28 & & $*$ Sm II & 6707.779 & 2.037 & -2.68 & \\
\hline Pr III & 6706.705 & 0.550 & -1.64 & DREAM & ${ }^{7} \mathrm{Li} \mathrm{I}$ & 6707.907 & 0.000 & -0.932 & [Smith et al.] \\
\hline *Nd II & 6706.738 & 2.868 & -2.48 & & ${ }^{7} \mathrm{Li} \mathrm{I}$ & 6707.908 & 0.000 & -1.161 & -"- \\
\hline *Sm II & 6706.789 & 1.586 & -2.00 & & ${ }^{7} \mathrm{Li} \mathrm{I}$ & 6707.919 & 0.000 & -0.712 & -"- \\
\hline *Sm II & 6706.807 & 1.874 & -1.78 & & ${ }^{6} \mathrm{Li} \mathrm{I}$ & 6707.920 & 0.000 & -0.478 & -"- \\
\hline Tm II & 6706.906 & 4.908 & -2.47 & DREAM & ${ }^{7} \mathrm{Li} \mathrm{I}$ & 6707.920 & 0.000 & -0.931 & $-"-$ \\
\hline *Nd II & 6706.922 & 3.211 & -0.88 & & ${ }^{6} \mathrm{Li} \mathrm{I}$ & 6707.923 & 0.000 & -0.179 & -"- \\
\hline *Nd II & 6707.015 & 1.490 & -1.88 & & Nd II & 6708.030 & 1.522 & -1.13 & DREAM[Quinet] \\
\hline$* \mathrm{Nd}$ II & 6707.033 & 2.222 & -3.68 & & ${ }^{6} \mathrm{Li} \mathrm{I}$ & 6708.073 & 0.000 & -0.304 & [Smith et al.] \\
\hline Ce II & 6707.121 & 1.255 & -3.76 & DREAM & $\mathrm{Ce}$ II & 6708.077 & 2.250 & -2.57 & DREAM \\
\hline *Dy II & 6707.153 & 3.292 & -1.27 & & $*$ Er II & 6708.088 & 3.155 & -2.58 & \\
\hline *Dy II & 6707.266 & 2.890 & -1.28 & & Ce II & 6708.099 & 0.701 & -2.12 & DREAM \\
\hline *Sm II & 6707.342 & 0.884 & -2.00 & & $* \mathrm{Nd}$ II & 6708.400 & 3.192 & -2.48 & \\
\hline$* \mathrm{Er}$ II & 6707.418 & 3.482 & -1.44 & & $* \mathrm{Nd}$ II & 6708.458 & 3.536 & -1.08 & \\
\hline$* \mathrm{Nd}$ II & 6707.433 & 1.499 & -2.17 & & $* \mathrm{Nd}$ II & 6708.629 & 0.746 & -4.58 & \\
\hline$* \mathrm{Nd}$ II & 6707.453 & 2.880 & -3.18 & & & & & & \\
\hline
\end{tabular}

${ }^{*} g f$-values were estimated by us from best fitting with observed spectrum.

allows us to select the elemental abundances automatically, and applying it to the available spectral ranges (6120-6180, 6675-6735 $\AA$ ), one obtains results which are close to those of Cowley et al. (2000).

The line list for spectra calculations was compiled from the DREAM database (http://www.umh.ac.be/ astro/ dream.shtml) and VALD (Kupka et al. 1999). When there was a choice, the preference was given to $g f$ values from DREAM. The wavelength and $g f$-values for lithium lines $\left({ }^{7} \mathrm{Li}\right.$ and ${ }^{6} \mathrm{Li}$ ) were taken from the paper of Smith et al. (1998). In addition, we have calculated wavelengths of REE lines, singly and doubly ionised, using the data of REE energy levels from the NIST database (2001) according to selection rules $(\delta J=0$, \pm 1 ) and combining even and odd levels of energy (odd-even or even-odd). All possible transitions between REE energy levels of NIST were included in the line list for synthetic spectra calculations. For lines additional to VALD and DREAM which are rather distant from $\mathrm{Li}$ lines and do not contribute noticeably to the $6708 \AA$ blend (see Table 4), $g f$-values were matched for better agreement of observed and calculated spectra.

$g f$-values for the two lines nearest to the lithium lines (Tables 2, 4), Nd II 6707.755 $\AA$ and Nd II 6708.030 were estimated by P. Quinet with Cowan's code using for the second line the trial identification of the top level, which was unspecified in the NIST list of REE energy levels. This line, Nd II $6708.030 \AA$, forms a blend with the strong line Ce II $6708.099 \AA$, whose $g f$ value is contained in the
DREAM database. In the region studied, there is a rather pure line Ce II 6706.051 $\AA$. The DREAM value of $\log g f=-0.95$ for this line provides Ce abundance $\log N(\mathrm{Ce})=-7.63$, which almost coincides with the estimate of Cowley et al. (2000), $\log N(\mathrm{Ce})=-7.60$. This $\mathrm{Ce}$ abundance value results in an estimated $\log N(\mathrm{Nd})=-7.97$ from the line $\mathrm{Nd}$ II $6708.030 \AA$ with $\log g f=-1.13$. This estimate differs from the value of Cowley et al. (2000) $\log N=-7.65( \pm 0.28)$ by 0.32 dex, but it only slightly exceeds the error value. Therefore, Quinet's estimate for the $g f$ value of $\mathrm{Nd}$ II 6708.030 seems a reasonable one. However, its blending with the ${ }^{6} \mathrm{Li}$ lines leads to some uncertainty in the estimation of the lithium isotopic ratio. The line of Sm II, $\lambda$ 6707.779, almost coincides with the centre of the lithium blend, but unfortunately, the calculations for Sm II are not possible because the energy matrix dimensions exceed the permitted sizes for Cowan's code. We tried to calculate the blend $6708 \AA$ with Sm II line absorption $\lambda$ 6707.779, instead of Li lines, adjusting the $g f$ value for better agreement with the observed blend. In this way we could estimate an upper limit to $\log g f$ for this line, -0.63 . The resulting fit was bad (see Fig. 2). Only the inclusion of ${ }^{7} \mathrm{Li}$ and ${ }^{6} \mathrm{Li}$ lines (see Table 2) with the ratio ${ }^{6} \mathrm{Li} /{ }^{7} \mathrm{Li}=0.3$ allowed us to represent the observed profile rather well (see Fig. 2). Our estimate of $\log g f$ for the Sm II line $\lambda 6707.779$ in this case is equal to -2.68 . We should repeat that our estimate of the lithium isotopic ratio is influenced by blending with the Nd II line 6708.030 ̊. 
Table 3. Element abundances for modelling of the 6705.75-6708.75 A spectrum.

\begin{tabular}{|c|c|c|c|c|c|}
\hline \hline El. & ${ }^{7} \mathrm{Li}$ only & \multicolumn{3}{|c|}{${ }^{6} \mathrm{Li} /{ }^{7} \mathrm{Li}=0.33$} & \\
\hline & $6600 / 4.2$ & $6600 / 4.2$ & $6750 / 4.0$ & $6600 / 4.2(\mathrm{PK})$ & $6600 / 4.2$ (Cowley et al. 2000) \\
\hline $\mathrm{Li}$ I & -8.90 & -8.90 & -8.80 & -8.83 & - \\
\hline $\mathrm{Ce}$ II & -7.63 & -7.63 & -7.65 & -7.63 & $-7.60+-0.26$ \\
\hline Pr III & -7.55 & -7.55 & -7.60 & -7.65 & $-7.46+-0.16$ \\
\hline $\mathrm{Nd}$ II & -7.97 & -8.07 & -7.87 & -8.07 & $-7.65+-0.28$ \\
\hline Sm II & -8.32 & -8.32 & -8.25 & -8.42 & $-7.75+-0.19$ \\
\hline
\end{tabular}

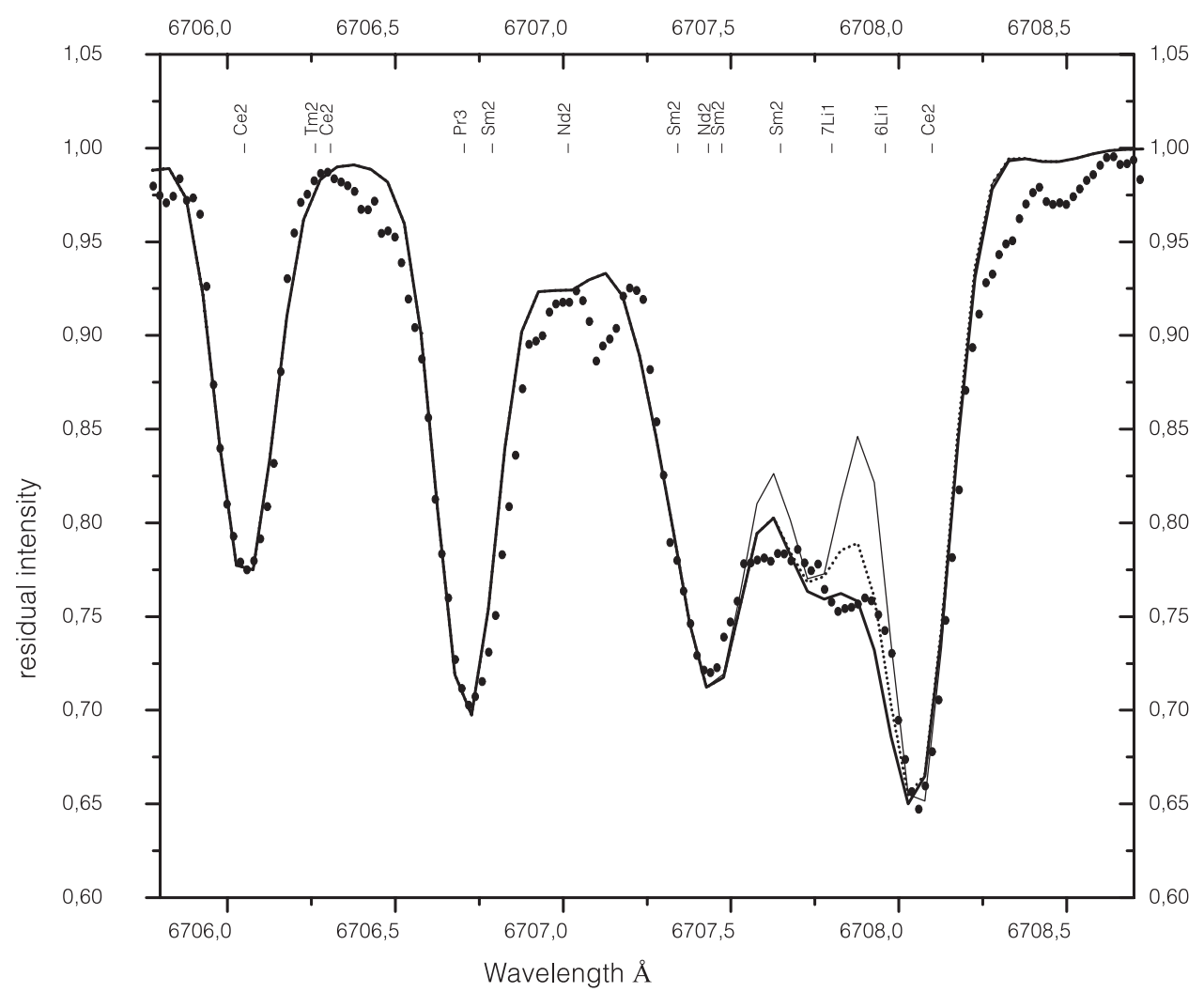

Fig. 2. The fitting of observed and calculated spectra of HD 101065 near $6708 \AA$ A: dots: observed spectrum; dashed line: calculated spectrum taking into account lines of the main isotope ${ }^{7} \mathrm{Li}$ only; thick line: spectrum with the ratio ${ }^{6} \mathrm{Li} /{ }^{7} \mathrm{Li}=0.3$. The thin line corresponds to a spectrum computed without Li but with the Sm II line $6707.799 \AA$. The positions of those lines which are the main contributors in absorption are marked at the top of the figure.

Table 2 contains the line list used in model calculations. The wavelengths of lines marked by an asterisk were calculated on the basis of NIST energy levels (2001). Corresponding $g f$-values were estimated by us using element abundances from Cowley et al. (2000), corrected for some REE elements (see Table 3 ) on the basis of lines with known $g f$-values (from DREAM and VALD lists). We used a value of microturbulent velocity $V_{\mathrm{t}}=2 \mathrm{~km} \mathrm{~s}^{-1}$ to compensate for magnetic line broadening. This value was selected by us for better agreement of computed and observed line profiles.

In Table 3 we present our results for Kurucz's and our models with $g f(\mathrm{REE}) * 10$ and the OS model (PK in the table) which is similar to that of Piskunov \& Kupka (2001), and compare them with data of Cowley et al. (2000) for $V_{\mathrm{t}}=2 \mathrm{~km} \mathrm{~s}^{-1}$. A better agreement was achieved for $\mathrm{Ce}$ II on the basis of several
Ce II lines with DREAM $g f$-values, the lithium abundances being almost the same for all models used in this work. We were forced to reduce the $\mathrm{Nd}$ II and $\mathrm{Sm}$ II abundances taken from Cowley et al. (2000) when we fitted the observed line profiles with theoretical ones. These values (see Table 3), based only on one line of each element, $6708.030 \AA$ of Nd II and $6707.473 \AA$ of Sm II, cannot be considered as reliable abundance estimates.

We should note that the distinctive feature of this star among other roAp stars is the predominance of numerous strong Ce II lines in the spectrum, which betrays the extreme overabundance of this ion. Not accounting for the DREAM line list of Ce II can result in overabundances for some REE and other elements.

In Table 4 we show the shares of the main contributors in total absorption (in percent) in each wavelength with 
Table 4. The main absorption contributors in the range 6707.60-6708.16 A.

\begin{tabular}{|c|c|c|c|c|c|c|c|c|c|c|c|c|c|c|c|c|c|c|c|c|c|c|c|c|c|c|c|c|c|c|c|}
\hline El. & $\lambda, \AA$ & .60 & .62 & .64 & .66 & .68 & .70 & .72 & .7 & .7 & & \begin{tabular}{l|l|}
78 \\
\end{tabular} & .80 & .82 & .84 & .86 & .88 & .90 & .92 & .94 & .96 & .98 & \begin{tabular}{|l|}
.00 \\
\end{tabular} & .02 & \begin{tabular}{|l|}
.04 \\
\end{tabular} & .06 & \begin{tabular}{|l|}
.08 \\
\end{tabular} & .10 & .12 & .14 & .16 \\
\hline Sm II & 6707.648 & 6 & 21 & 20 & 20 & 12 & 6 & 2 & 1 & & & & & & & & & & & & & & & & & & & & & & \\
\hline $\mathrm{Nd}$ II & 6707.755 & & & & & & 1 & 1 & 2 & 2 & 2 & 1 & 1 & & & & & & & & & & & & & & & & & & \\
\hline${ }^{7} \mathrm{Li} \mathrm{I}$ & 6707.756 & 1 & 2 & 3 & 3 & 4 & 5 & 6 & 7 & r & 7 & 6 & 6 & 5 & 4 & 2 & 1 & 1 & 1 & & & & & & & & & & & & \\
\hline${ }^{7} \mathrm{Li} \mathrm{I}$ & 6707.768 & 1 & 3 & 4 & 4 & 5 & 7 & 9 & 1 & 1 & 11 & 11 & 10 & 9 & 7 & 4 & 3 & 2 & 2 & 1 & & & & & & & & & & & \\
\hline${ }^{7} \mathrm{Li} \mathrm{I}$ & 6707.907 & & & & & & & & & & & & 1 & 1 & 1 & 1 & 2 & 2 & 2 & 2 & 2 & 1 & 1 & 1 & & & & & & & \\
\hline${ }^{7} \mathrm{Li} \mathrm{I}$ & 6707.908 & & & & & & & & & & & & 1 & 1 & 1 & 1 & 1 & 1 & 1 & 1 & 1 & 1 & 1 & & & & & & & & \\
\hline${ }^{6} \mathrm{Li} \mathrm{I}$ & 6707.919 & & & & & & & & & & & 1 & 1 & 1 & 2 & 2 & 3 & 3 & 3 & 3 & 3 & 3 & 2 & 1 & 1 & & & & & & \\
\hline${ }^{7} \mathrm{Li} \mathrm{I}$ & 6707.920 & & & & & & & & & & & & 1 & 1 & 1 & 1 & 1 & 2 & 2 & 2 & 2 & 2 & 1 & 1 & & & & & & & \\
\hline${ }^{6} \mathrm{Li} \mathrm{I}$ & 6707.920 & & & & & & & & & & & & 1 & 1 & 1 & 1 & 2 & 2 & 2 & 2 & 2 & 2 & 1 & 1 & & & & & & & \\
\hline${ }^{6} \mathrm{Li} \mathrm{I}$ & 6707.923 & & & & & & & & & & & & 1 & 2 & 2 & 2 & 3 & 3 & 3 & 4 & 3 & 3 & 3 & 1 & 1 & 1 & & & & & \\
\hline Nd II & 6708.029 & & & & & & & & & & & & & & & & & & & 2 & 8 & 26 & 62 & 75 & 71 & 55 & 31 & 12 & 3 & & \\
\hline${ }^{6} \mathrm{Li} \mathrm{I}$ & 6708.073 & & & & & & & & & & & & & & & & & & & 1 & 1 & 2 & 2 & 2 & 3 & 3 & 4 & 4 & 3 & 2 & 1 \\
\hline Ce II & 6708.077 & & & & & & & & & & & & & & & & & & & & & & & & & 1 & 1 & 1 & & & \\
\hline $\mathrm{Ce}$ II & 6708.099 & & & & & & & & & & & & & & & & & & & & & & 1 & 3 & 8 & 21 & 37 & 46 & 28 & 13 & 5 \\
\hline
\end{tabular}

a $0.02 \AA$ step, as they are calculated by the STARSP code, for the lithium doublet range 6707.60-6708.16 $\AA$ without instrumental smoothing, for the model atmosphere 6600/4.2 with $V_{\mathrm{t}}=2 \mathrm{~km} \mathrm{~s}^{-1}$ and ${ }^{6} \mathrm{Li} /{ }^{7} \mathrm{Li}=0.3$.

Reyniers et al. (2002) draw attention to a possible error in Li line identification (instead of the Ce II line 6708.03) in the spectra of s-process enriched post-AGB stars. In our high resolution spectra, these two lines are sufficiently far apart to be distinguished, as can be seen in Fig. 2 and Table 4.

\section{Discussion}

Since the discovery of the star HD 101065 by Przybylski, the debates relating to the effective temperature of this star have not settled yet. Apparently, the reason lies in the large blanketing effect due to strong absorption in REE lines, identified and unidentified. Incompleteness of data for these lines does not allow one to take into account correctly this effect in model atmosphere calculations. Cowley et al. (2000) have clearly shown a large excess of REE abundances (4 dex) and deficiency of iron peak elements $(\mathrm{Fe}, \mathrm{Ni})$, while $\mathrm{Co}$ has appeared in excess of 1.5 dex. The list of REE lines in this work included new identified lines in the spectral range 3959-6652 A. Our results include the range 6670-6735 $\AA$ where lies the remarkable feature with the resonant lithium doublet line $6708 \AA$.

We have carried out detailed calculations of the blend $6708 \AA$ (6705.75-6708.75 $\AA$ ), using the REE list of atomic data from the DREAM and VALD databases. The evidence for the presence of lithium in the spectrum of HD 101065 is most probable, as shown by the excellent fit of synthetic spectra which include the Li lines, while the synthetic spectra without lithium (only REE lines, Sm II 6707.799) clearly fail to achieve a good match. All possible transitions between REE energy levels of NIST were included in the line list for synthetic spectra calculations. The presence of other REE lines near $6708 \AA$ that would not be included in our list remains improbable. All transitions near $6708 \AA$ are due to low energy levels, and low energy REE levels that would not belong to NIST are unlikely. The only line which might mimic the Li lines is Sm II 6707.799 A. But we showed that absorption in this line is not large enough to account for the observed spectral feature, while a perfect fit is obtained when accounting for the Li line components, which are distributed over rather wide range (see Tables 2 and 4).

In our work the choice of parameters of the synthetic spectrum near the line Li I $6708 \AA$ is described in detail taking into account the REE lines with known and unknown $g f$ values. The variants of the calculations, with and without lithium lines, are considered, which prove the presence of a lithium doublet $6708 \AA$ in the spectrum of HD 101065 with a lithium abundance of 3.1 dex (in the scale $\log N(\mathrm{H})=12.0$ ). The observed blend is best represented at the isotopic ratio ${ }^{6} \mathrm{Li} /{ }^{7} \mathrm{Li}=0.3$. The latter value can be considered as an upper limit in the current state of atomic data; more accurate estimation of the lithium isotopic ratio would require better spectral resolution and better $g f$-values for REE lines.

We carried out the calculations with Zeeman and PaschenBack splitting of the Li line. For $H=2300 \mathrm{G}$, the PaschenBack effect is negligible (this is common for magnetic fields lower than $4000 \mathrm{G}$ - see Shavrina et al. 2000) and cannot induce any additional asymmetry. Zeeman broadening is also unimportant, due to the significant separation $(0.15 \AA)$ of the components of the Li doublet. On the basis of our spectra calculations in two wider spectral ranges, 6120-6180 $\AA$ and 6675-6735 $\AA$, the value $V_{\mathrm{t}}=2 \mathrm{~km} \mathrm{~s}^{-1}$ was adopted, which must compensate for the magnetic broadening of other lines. We have also considered the possible binarity of HD 101065. This problem was discussed by Hubrig et al. (2000). They noticed that none of the 14 roAp considered, including HD 101065, is known to be a spectroscopic binary (although several are members of wide visual binary systems); therefore 
the $6708 \AA$ feature cannot be due to a secondary component of this star.

Warner's (1966) estimate of the lithium abundance for this star from a spectrum with $0.3 \AA$ resolution was 2.4 dex relative to the solar value, that is, 3.3 dex relative to hydrogen. $\mathrm{He}$ also assumed the presence of ${ }^{6} \mathrm{Li}$ on the surface of the star. The good agreement between our results and those of Warner (1966) is encouraging. Warner had tried to explain the large excess of rare earth elements and the peculiar structure of the atmosphere by dragging out r-process elements from deeper to surface layers of the star. However, the high lithium content observed, $3.1 \mathrm{dex}$ (on the scale where $\log N(H)=12$ ), is not unexpected for normal $\mathrm{F}$ stars, which are separated into high and low Li abundances (Chen et al. 2001), while Warner's assumption would imply complete mixing and, therefore, complete lithium destruction. Thus the radiative diffusion mechanism seems a much more promising explanation, especially as the magnetic field, reaching $2300 \mathrm{G}$ (Cowley et al. 2000), would make it easier. On the other hand, the isotopic ratio ${ }^{6} \mathrm{Li} /{ }^{7} \mathrm{Li}=0.3$ might be considered as indicating spallation reactions on the stellar surface, though a combination of radiative and ambipolar diffusion might perhaps lead to the same result.

The Hipparcos measurements have confirmed that the star is a dwarf or a subgiant: using its parallax (7.95 \pm 1.07 mas) and the adopted $T_{\text {eff }}=6750 \mathrm{~K}$, we obtain $\log g=4.00 \pm 0.13$ by interpolation in evolutionary tracks, assuming a normal evolution and a solar global composition. However, its spectrum is reminiscent of S-stars (except for Li!), whose atmospheres are enriched by freshly synthesized material from deep layers. Whether the extreme anomaly of the spectrum of this interesting star is just another case of radiative diffusion, or requires a completely new explanation, remains to be seen.

Acknowledgements. The data from Kurucz's CDROM 23, NASA ADC, VALD2, NIST and DREAM, were used and we thank the administrations of these databases who provided access to the data via INTERNET.

N. S. Polosukhina thanks the Consortium of Physics of the Trieste University for financial support. A.Yushchenko was supported by the grant of Post-Doc Program, Chonbuk National University (2002). We thank our referee, Dr. M. Gerbaldi, for valuable critical comments that have helped us to improve the paper.

\section{References}

Asplund, M., Gustafsson, B., Lambert, D. L., \& Rao, N. K. 2000, A\&A, 353, 287

Biémont, E., Palmeri, P., \& Quinet, P., D.R.E.A.M. Database on Rare Earth at Mons.Univ., http://www.umh.ac.be/ astro/ dream.shtml
Chen, Y. Q., Nissen, P. E., Benoni, T., \& Zhao, G. 2001, A\&A, 371, 943

Cowley, C. R., \& Mathys, G. 1998, A\&A, 339, 165

Cowley, C. R., Ryabchikova, T., Kupka, F., et al. 2000, MNRAS, 317 , 299

Hofsaess, D. 1979, Atom. data. Nucl. data Table, 24, 285

Hubrig, S., Kharchenko, N., Mathys, G., \& North, P. 2000, A\&A, 355, 1031

Kupka, F., Piskunov, N. E., Ryabchikova, T. A., Stempels, H. C., \& Weiss, W. W. 1999, A\&AS, 138, 119

Kurtz, D., \& Wegner, G. 1979, ApJ, 232, 510

Kurucz, R. L. 1993, CD ROM N 1-23 (Cambridge, MA: Smithsonian Astrophys. Obs.)

Kurucz, R. L. 1970, Smithsonian Astrophys. Obs. Spec. Rept. N, 309, 1

Kurucz, R. L. 1999, http://cfaku5 . harvard.edu/

Matthews, J. M., Kurtz, D. V., \& Martinez, P. 1999, ApJ, 511, 422

Meggers, W. F., Corliss, C. H., \& Seribnez, B. F. 1961, Nat. Bur. Stand., Mon. 32

North, P., Polosukhina, N., Malanushenko, V., \& Hack, M. 1998, A\&A, 333, 644

Martin, W. C., Sugar, J., \& Musgrove, A. 2001, Energy Levels Database, http://physics.nist.gov/cgi-bin/AtData

Pavlenko, Ya. V. 1999, Astron. Rept., 43, 94

Pavlenko, Ya. V, \& Zhukovskaya, S. 2003, Kinematics and Physics of Celestial bodies, 19, 29

Pavlenko, Ya. V. 2003a, Proc. of IAU 210, Uppsala, ed. N. Piskunov, in press [astro-ph/0209022]

Pavlenko, Ya. V. 2003b, Astron. Rept., 47, 59

Pavlenko, Ya. V. 2003c, http://www.mao.kiev.ua/staff/yp/ Results

Piskunov, N., \& Kupka, F. 2001, A\&A, 547, 1040

Przybylski, A. 1961, Nature, 189, 739

Przybylski, A. 1966, Nature, 210, 20

Quinet, P., Palmeri, P., \& Biémont, E. 1999, JQSRT 62, 625

Reyniers, M., Van Winckel, H., Biémont, E., \& Quinet, P. 2002, A\&A, L395

Seaton, M. J. 1992, Rev. Mex. Astron. Astrofis., 23, 180

Shavrina, A. V., Polosukhina, N. S., Tsymbal, T., \& Khalack, V. R. 2000, Astron. Rept., 44, 235

Shavrina, A. V., Polosukhina, N. S., Zverko, J., et al. 2001, A\&A, 372, 571

Smith, V. V., Lambert, D. L., \& Nissen, P. E. 1998, ApJ, 506, 405

Sneden, C., Johnson, H., \& Krupp, B. 1976, ApJ, 204, 281

Tsymbal, V. 1996, M.A.S.S., ed. S. Adelman, F. Kupla, \& W. W. Weiss, ASP Conf. Ser., 108, 198

Unsold, A. 1955, Physics der Sternatmospharen, 2nd ed. (Berlin: Springer)

Yushchenko, A. V. 1997, Proc. of the 20th Stellar Conf. of the Czech and Slovak Astronomical Institutes, Brno, Czech Republic, ed. J. Dusek. ISBN 80-85882-08-6. Brno 1998, 201

Warner, B. 1966, Nature, 211, 55

Wegner, G., \& Petford, A. D. 1974, MNRAS, 168, 575

Wolff, S. C., \& Hagen, W. 1976, PASP, 88, 119 